\title{
GUIDELINES
}

\section{Management of lower urinary tract symptoms in men: summary of NICE guidance}

\author{
Clare Jones, ${ }^{1}$ Jennifer Hill, ${ }^{1}$ Christopher Chapple, ${ }^{12}$ on behalf of the Guideline Development Group
}

${ }^{1}$ National Clinical Guideline Centre for Acute and Chronic Conditions, Royal College of Physicians, London NW1 4LE

${ }^{2}$ Department of Urology, Royal Hallamshire Hospital, Sheffield Teaching Hospitals NHS

Foundation Trust, Sheffield S10 2JF

Correspondence to: C Chapple c.r.chapple@sheffield.ac.uk

Cite this as: BMJ 2010;340:C2354 doi: 10.1136/bmj.c2354

This is one of a series of $B M J$ summaries of new guidelines based on the best available evidence; they highlight important recommendations for clinical practice, especially where uncertainty or controversy exists The supporting evidence statements and further information about the guidance are in the full version on bmj.com.
Lower urinary tract symptoms (LUTS) in men are related to problems with storage and voiding of urine and can occur after micturition. The many causes of LUTS include abnormalities or abnormal function of the bladder, prostate, urethra, or sphincters. The prevalence of LUTS increases as men get older, with about $30 \%$ of men aged 65 years and older having troublesome symptoms, ${ }^{1}$ which may require treatment. This article summarises the most recent recommendations from the National Institute for Health and Clinical Excellence (NICE) on LUTS. $^{2}$

\section{Recommendations}

NICE recommendations are based on systematic reviews of best available evidence and explicit consideration of cost effectiveness. When minimal evidence is available, recommendations are based on the guideline development group's experience and opinion of what constitutes good practice. Evidence levels for the recommendations are in the full version of this article on bmj.com.

Lower urinary tract symptoms have been categorised according to the three stages of the bladder cycle ${ }^{3}$ : storage (when filling of the bladder occurs), voiding (when the bladder actively expels its contents), and post micturition (immediately after voiding, while returning to the storage stage) (box).

\section{Initial assessment}

At initial assessment (in any setting and by a healthcare professional without specific training in managing LUTS in men) take the following actions.

- Offer the patient an assessment of his general medical history to identify possible causes of LUTS and associated comorbidities; review current medication, including herbal and over the counter medicines (for example, Serenoa repens (saw palmetto) and pumpkin seed extract), to identify drugs that may be contributing to the problem.

- Offer a physical examination guided by urological symptoms and other medical conditions; an examination of the abdomen and external genitalia; and a digital rectal examination.

- Ask men with symptoms that interfere with quality of life to complete a "urinary frequency/volume chart," recording the frequencies and volumes of fluid drunk and voided. ${ }^{4}$
- Offer a urine dipstick test to detect blood, glucose, protein, leucocytes, and nitrites.

- Offer the patient information and advice, and give him the time to decide whether he wishes to have prostate specific antigen testing if:

-His symptoms suggest bladder outlet obstruction secondary to benign prostatic enlargement or -His prostate feels abnormal on digital rectal examination or

-He is concerned about prostate cancer.

- Offer a serum creatinine test (plus calculation of estimated glomerular filtration rate) only if you suspect renal impairment (for example, if the man has a palpable bladder, nocturnal enuresis, recurrent urinary tract infections, or a history of renal stones).

- Refer for specialist assessment (in any setting by a healthcare professional with specific training in managing LUTS in men) if the symptoms are complicated by recurrent or persistent urinary tract infection, retention, renal impairment that is suspected to be caused by lower urinary tract dysfunction, or suspected urological cancer.

- Offer men considering any treatment for LUTS an assessment of their baseline symptoms with a validated symptom score (for example, the international prostate symptom score ${ }^{56}$ ) to allow assessment of subsequent changes in symptoms.

Categories of lower urinary tract symptoms ${ }^{3}$
Storage (also symptoms of overactive bladder)
Urgency
Increased daytime frequency
Nocturia
Urinary incontinence
Voiding
Hesitancy
Straining
Slow stream
Splitting or spraying
Intermittency
Terminal dribble
Post-micturition
Post-micturition dribble
Feeling of incomplete emptying




bmj.com archive
Previous articles in this
series
Diagnosis and
management of idiopathic
childhood constipation
(BMJ 2010;340:c2585)
Neonatal jaundice
(BMJ 2010;340:c2409)
Pharmacological
management of
neuropathic pain in non-
specialist settings
(BMJ 2010;340:c1079)
- Assessment of recent
onset chest pain or
discomfort of suspected
cardiac origin
(BMJ 2010;340:c1118)
E Early management
of unstable angina and
non-ST segment elevation
myocardial infarction
(BMJ2010;340:c1134)

Conservative management

- Offer men with storage symptoms (particularly urinary incontinence) temporary containment products (for example, pads or collecting devices) so that they can contuinue their normal daily activities until a diagnosis and management plan have been discussed.

- If symptoms are not bothersome or complicated, reassure the patient by explaining the possible causes of these symptoms, offer advice on lifestyle interventions (for example, adjusting fluid intake) and information on their condition. Offer a review if symptoms change.

- For men with symptoms suggestive of an overactive bladder (see box), offer supervised bladder training (which aims to reduce urgency and urinary frequency through a gradual increase in the intervals between urination episodes), advice on fluid intake, lifestyle advice (for example, caffeine and alcohol reduction), and if needed containment products.

\section{Drug treatment}

- For men with moderate to severe symptoms, offer an $\alpha$ blocker (alfuzosin, doxazosin, tamsulosin, or terazosin).

- For men with symptoms of an overactive bladder, offer an anticholinergic.

\section{Specialist assessment}

Specialist assessment refers to the assessment carried out in any setting by a healthcare professional with specific training in managing LUTS in men.

- For men with complicated symptoms or bothersome symptoms that have not responded to conservative management or drug treatment, offer referral for specialist assessment.

- Offer cystoscopy to those having specialist assessment only when clinically indicated-for example, if the patient has a history of recurrent infection, sterile pyuria, haematuria, severe symptoms, or pain.

- Offer imaging of the upper urinary tract to those having specialist assessment only when clinically indicated-for example, if the patient has a history of chronic retention, haematuria, recurrent infection, sterile pyuria, severe symptoms, or pain.

\section{Surgery for voiding symptoms}

- If offering surgery for managing voiding symptoms that are presumed secondary to benign prostatic enlargement, offer monopolar or bipolar transurethral resection of the prostate (TURP), monopolar transurethral vaporisation of the prostate (TUVP), or holmium laser enucleation of the prostate (HoLEP). Perform HoLEP at a centre that specialises in the technique or has mentorship arrangements.

- If offering surgery for managing voiding symptoms that are presumed secondary to benign prostatic enlargement, do not offer minimally invasive treatments (including transurethral needle ablation, transurethral microwave thermotherapy, high intensity focused ultrasound, transurethral ethanol ablation of the prostate, and laser coagulation) as alternatives to TURP, TUVP, or HoLEP, as there is insufficient evidence that these techniques offer any advantage over TURP.

\section{Providing information}

- Make sure men with LUTS have access to care that can help with their emotional and physical conditions and with relevant physical, emotional, psychological, sexual, and social issues.

- Advise men with storage symptoms (particularly incontinence) about relevant support groups.

\section{Overcoming barriers}

Lower urinary tract symptoms (storage, voiding, and postmicturition) can affect both men and women (see the recent NICE guidance on female incontinence ${ }^{7}$ ), and this guidance on male LUTS aims to dispel the stereotype that men have only obstructive symptoms whereas women have incontinence. Men tend to be slow to seek health advice, particularly with symptoms that may be considered sensitive. This guidance is aimed at ( $a$ ) encouraging healthcare practitioners to recognise the impact of LUTS on quality of life; (b) overcoming any stigma related to these symptoms; and (c) highlighting for patients and healthcare professionals alike that symptoms can be managed effectively after simple initial assessment. To enable accurate categorisation of underlying problems of the lower urinary tract, the guidance recommends the routine use of a simple chart for recording the frequencies and volumes of fluids drunk and voided. Use of the chart may show, for example, that urinary frequency is associated with drinking too much fluid or with underlying comorbidity, rather than being caused by a disorder of the lower urinary tract.

Primary care lacks resources for managing lower urinary tract symptoms, with not enough healthcare professionals specialising in this area. One way to overcome this could be to change reimbursement strategies, such as the quality and outcomes framework, to increase the incentives to improve care.

Contributors: All authors contributed to a first draft and shared the subsequent editing. CC is guarantor.

Funding: The National Clinical Guideline Centre for Acute and Chronic Conditions was commissioned and funded by the National Institute for Health and Clinical Excellence to write this summary.

Competing interests: All authors have completed the Unified Competing Interest form at www.icmje.org/coi_disclosure.pdf (available on request from the corresponding author) and declare (1) $\mathrm{JH}, \mathrm{CJ}$ and CC have support from NICE for work on guideline development: $\mathrm{JH}$ and her department are funded by NICE, and CC received honorariums for chairing the guideline development meetings; (2) CJ and JH have no relationships with any company that might have an interest in the submitted work; CC has consultancy relationships with Pfizer, Astellas, Novartis, Tanabe, Recordati, ONO, Xention and Allergan; grant relationships with Pfizer and Allergan; trial participation relationships with Pfizer, Astellas, Tanabe, Recordati, and Allergan that might have an interest in the submitted work in the previous 3 years (3) no spouses, partners, or children have financial relationships that may be relevant to the submitted work; and (4) no non-financial interests that may be relevant to the submitted work.

Provenance and peer review: Commissioned; not externally peer reviewed. 
1 Chapple CR. BPH disease management. Introduction and concluding remarks. Eur Urol 1999;36(suppl 3):1-6.

2 National Institute for Health and Clinical Excellence. The management of lower urinary tract symptoms in men. (Clinical guideline 97.) 2010. www.nice.org.uk/CG97

3 Abrams P, Cardozo L, Fall M, Griffiths D, Rosier P, Ulmsten U, et al. The standardisation of terminology of lower urinary tract function: report from the Standardisation Sub-committee of the International Continence Society. Neurourol Urodyn 2002;21:167-78.

4 Chapple CR, MacDiarmid SA, Patel A. Urodynamics made easy. 3 rd ed. Churchill Livingstone, 2009.
5 Barry MJ, Fowler FJ Jr, O'Leary MP, Bruskewitz RC, Holtgrewe HL, Mebust WK, et al. The American Urological Association symptom index for benign prostatic hyperplasia. The Measurement Committee of the American Urological Association. J Urol 1992;148:1549-57.

6 Mebust WK, Roizo R, Schroeder F, Villers A. Correlations between pathology, clinical symptoms and the course of the disease. International Consultation on Benign Prostatic Hyperplasia Proceedings 1991:51-62.

7 National Institute for Health and Clinical Excellence. Urinary incontinence: the management of urinary incontinence in women. (Clinical guidance 40.) 2006. www nice org uk/CG40.

\title{
DIAGNOSIS IN GENERAL PRACTICE When no diagnostic label is applied
}

\author{
Roger Jones, ${ }^{1}$ Kevin Barraclough, ${ }^{2}$ Christopher Dowrick ${ }^{3}$
}

$\overline{{ }^{1} \text { King's College London, London }}$
SE13QD
${ }^{2}$ Painswick GL6 6TY
${ }^{3}$ University of Liverpool

University of Liverpool

Correspondence to: $\mathrm{R}$ Jones

roger.jones@kcl.ac.uk

Cite this as: BMJ 2010;340:c2683 doi: 10.1136/bmi.c2683

This series aims to set out a diagnostic strategy and illustrate its application with a case. The series advisers are Kevin Barraclough, general practitioner, Painswick, and research fellow in community based medicine, University of Bristol; Paul Glasziou, professor of evidence based medicine, Department of Primary Health Care, University of Oxford; and Peter Rose, university lecturer, Department of Primary Health Care, University of Oxford.

\section{Resisting the temptation to attach a diagnostic label to indeterminate symptoms can be in the patient's interests}

\section{Case scenario}

A 38 year old woman presented with "indigestion type" symptoms. She had a long history of frequent visits to her general practitioner (GP) and investigations for unexplained pelvic pain, dizziness, and headache. On this occasion she felt a "burning" sensation "in a band across my tummy" and felt as though the ground was unsteady when she walked. Her GP could reach no diagnosis and said he believed her symptoms were likely to resolve, advising follow-up in a few weeks.

\section{Diagnostic labels}

A “diagnostic label” in general practice is generally a working diagnosis that functions as a decision node. It is different from the histologically or microbiologically proved diagnoses made in secondary care and tends to take one of three forms:

- A working diagnosis on which treatment is based (such as "acute otitis media")

- A working diagnosis on which further investigations are planned (such as "bloody diarrhoea?

inflammatory bowel disease")

- A working diagnosis indicating the absence of serious disease (such as “calf pain, not DVT”).

The aim of this article is to understand why diagnostic labels are sometimes not applied in general practice consultations and to understand how this approach contributes to making decisions about diagnoses.

\section{KEY POINTS}

Some patients who present in primary care cannot be given a definite diagnostic label Not giving a diagnostic label may indicate to the patient that nothing serious is wrong or may leave the door open for further investigation and clarification of symptoms

A definite, final diagnosis cannot be made in a large proportion of patients presenting with physical symptoms

Patients falling into the spectrum of medically unexplained physical symptoms (MUPS) are likely to require particularly skilful management

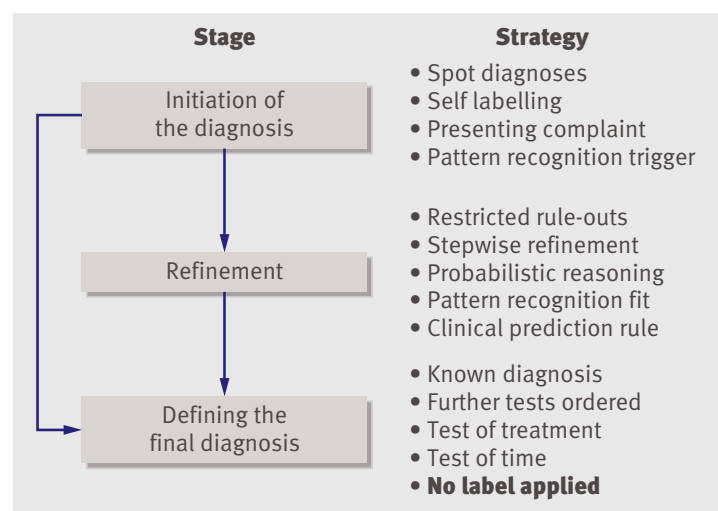

Stages and strategies in arriving at a diagnosis

\section{Why is a diagnosis sometimes missing?}

Bruce Thomas, a general practitioner in Hampshire, UK, pointed out in the 1970 s that in up to $40 \%$ of patients in general practice no diagnostic label could be attached. ${ }^{12}$ These patients did not require, and generally were not given, specific treatment, and most recovered spontaneously. This remains the case. In a 2005 review of consultation data in the UK, "symptoms, signs and ill-defined conditions" was the computer coded group of disorders presenting most frequently in general practice. ${ }^{3}$

Also in the 1970s, Howie described "the relative rarity of fully developed hospital illness" in general practice. He pointed out that using the same diagnostic terminology in general practice as was used in hospitals could sometimes be misleading because of the "different spectrum of disease" and the different level of diagnostic certainty in primary care. ${ }^{4}$ In primary care, diagnostic labels can give spurious and erroneous diagnostic precision when what is being managed is what Howie described as a symptom:sign complex (such as non-cardiac chest pain with mild localised chest wall tenderness).

\section{When is management without a diagnostic label used?}

For many patients in general practice, the diagnostic strategies described by Heneghan et al (figure) are appropriate. ${ }^{5}$ When pattern recognition and iterative diagnosis ${ }^{6}$ do not give an answer, when symptoms are vague, or when a clear diagnosis does not "crystallise" from investigations or tests 
of time or of treatment, patients will need to be managed without having a diagnostic label. In not applying a diagnostic label the clinician may be "leaving the door open" for further investigations if symptoms persist. Alternatively, the lack of a label may be part of letting the patient know that, as far as can be ascertained, their symptoms do not add up to anything substantial or serious. Many patients to whom no diagnostic label can be attached can be managed without treatment or further investigation.

In the 1980s Thomas examined patient satisfaction in 200 consultations with patients who had symptoms but no abnormal physical signs. The patients were randomised to receive either a "positive" consultation (when they were given a firm diagnosis and told they would be better in a few days) or a "negative" consultation (in which no specific diagnosis or assurance was given), and also to receive either thiamine hydrochloride as a placebo or no treatment. Two weeks after the consultation $64 \%$ of those who were managed with a positive consultation reported being recovered as opposed to $39 \%$ of those given no assurances. ${ }^{7}$ The placebo drug treatment made little difference. This is consistent with a recent review of the placebo effect, which attributed the efficacy of the placebo to the overall content of the therapeutic interaction (doctor, patient, and context) rather than to any psychological effect that is specific to the (therapeutically inert) pill. ${ }^{8}$ Thomas's study suggests that taking a positive attitude in the consultation may accelerate patients' spontaneous recovery from a vague symptom complex. Many patients without an obvious diagnostic label could be managed in this way.

The remaining patients, those with persistent symptoms still requiring investigation, contain three subgroups:

- Those with important diagnoses that have not yet been made, which may be rare or are easily missed, ${ }^{9}$ such as Addison's disease, coeliac disease, and cancer of the ovary or pancreas

- Those with new or emerging conditions, such as obstructive sleep apnoea or chronic cough from gastrooesophageal reflux disease (many of whom were previously labelled as having "psychogenic cough")

- Those with persisting symptoms whose physical basis is unclear.

The last (and largest) group will often have been extensively investigated for poorly defined, often painful, conditions that are often associated with apparently disproportionate effects on mood and general wellbeing. These

Causes of wrong diagnoses in primary care

Cognitive oversight-simply not thinking of the correct diagnosis ${ }^{14}$-for example, forgetting coeliac disease as a cause of iron deficiency anaemia

Failure to gather adequate data-for example, inadequate physical examination for lower bowel symptoms caused by colorectal cancer

Misinterpretation of data-for example, diagnosing gout on the basis of a raised serum urate concentration or excluding it on the basis of normal serum urate.

Anchoring-sticking to an initial diagnosis despite disconfirming evidence, ${ }^{15}$ such as treating fatigue as depression despite evidence of abnormal renal function

Inappropriate confirmation-selective use of evidence to confirm an incorrect diagnosis, such as attributing importance to minor abnormalities in laboratory tests as an explanation for fatigue in someone with depression

Premature closure-arriving at a conclusive diagnosis before collecting all the data, ${ }^{16}$ such as diagnosing intermittent (vascular) claudication in a patient with lumbar canal spinal stenosis include patients with so called medically unexplained physical symptoms (MUPS), which comprise conditions such as dizziness, temporomandibular joint dysfunction, fibromyalgia, pelvic pain, "functional” abdominal pain, dyspepsia, and non-cardiac, non-reflux chest and thoracic pain. These patients present particular challenges for long term management, because they generally attend often and generate high healthcare costs, through over-investigation and inappropriate referral. From time to time clinicians may encounter patients with definite physical symptoms, such as haematuria or haemoptysis, in whom no definite diagnosis can be made. In a cohort study of patients with alarm symptoms in primary care, GPs did not make a diagnosis in over $60 \%$ of 37000 patients. $^{10}$

\section{Case study}

The following week, the 38 year old patient consulted her out of hours service and was given a prescription for a proton pump inhibitor for a presumed diagnosis of indigestion. Next morning she telephoned the surgery asking for a sick note as she was unable to get to work.

\section{How does diagnosis go wrong?}

Misdiagnosis can occur in two directions. Firstly, the doctor may conclude incorrectly that a patient does not have a condition to which a diagnostic label should be attached, or for which treatment is needed, and hence allow undiagnosed symptoms to persist, such as gastro-oesophageal reflux causing "non-cardiac" chest pain or chronic cough. Conversely, the doctor may confer a formal diagnosis on the patient's symptoms, despite the absence of adequate evidence of a recognisable medical disorder, such as making a diagnosis of vertebrobasilar insufficiency in a patient with vague unsteadiness. A sense of pressure to make a specific diagnosis can come from patients, from fear of litigation if a disease is missed, ${ }^{11}$ or from the expected role of general practitioners as identifiers of disease. $^{12}$

Wrong diagnoses in primary care can be caused by atypical and non-specific presentations, very rare conditions, and comorbidity, and also perceptual factors such as failure to observe subtle changes in colour or contour or abnormal swellings ${ }^{13}$ or gradual changes over time, as sometimes happens with patients with hypothyroidism or acromegaly. Other causes are shown in the box.

\section{How can we improve?}

Clinicians need to understand the various ways in which diagnostic labels should and should not be applied to patients in general practice, and they should be prepared to deal with the potential causes of diagnostic error listed in the box. This has important implications for medical education: performing clinical investigations and looking for symptoms and signs need to be taught with a probabilistic approach to differential diagnoses. Spuriously accurate clinical diagnoses need to be discouraged, keeping in mind that patients benefit from a certainty of approach.

Managing unexplained symptoms requires a high quality relationship between doctor and patient, characterised by a sympathetic and empathic inquiry into the psychosocial background of symptoms as well as the provision of considerable emotional support. Patients with medically 
unexplained symptoms are no more likely than those with explained symptoms to seek explanation, reassurance, or somatic interventions such as special investigations, drug treatment, or specialist referral-but they are much more likely to seek emotional support from their clinician. ${ }^{17}$ They are also more likely to be cautious about the extent to which clinicians will take their problems seriously. ${ }^{1819}$ Clinicians may manage these patients more effectively if they explain the limited, and potentially negative, value of repeat investigations, and if they avoid premature closure by regularly reviewing previous diagnostic labels or apparent lack of such labels. Clinicians may avoid unfounded diagnoses and subsequent somatic interventions by getting patients to talk about their social and psychological problems. ${ }^{2021}$ Brief educational interventions, including didactic sessions, small group work, and role play with video feedback can increase GPs' awareness of medically unexplained symptoms. ${ }^{22}$

\section{Case study}

The general practitioner asked the woman to return for review. A careful history elicited a description of a superficial "burning sensation" across the lower chest and increasing difficulty in walking. A Romberg test was positive, and she had increased tone and hyper-reflexia in her legs. Admission and magnetic resonance imaging of her neck showed a central cervical disc prolapse causing a myelopathy.

Contributors: RJ wrote the first draft of the paper, which was extensively modified by $C D$ and $K B$ before being completed by all the authors. $R$ J is guarantor. Competing interests: CD has two grants to study MUS in primary care with the Medical Research Council.

Provenance and peer review: Commissioned; externally peer reviewed.

1 Thomas KB. Temporarily dependent patient in general practice. BM] 1974;1:625-6.

2 Thomas KB. The consultation and the therapeutic illusion. BM 1978:1:1327-8

3 Fleming DM, Cross KW, Barley MA. Recent changes in the prevalence of diseases presenting for health care. BrJ Gen Pract 2005;55:589-95.
4 Howie JGR Diagnosis-the Achilles heel? IR Coll Gen Pract 1972:22:310.

5 Heneghan C, Glasziou P, Thompson M, Rose P, Balla J, Lasserson D, et al. Diagnostic strategies used in primary care. BMJ 2009;338:b946.

6 Norman G, Barraclough K, Dolovich L, Price D. Iterative diagnosis. BMJ 2009;339:b3490

7 Thomas KB. General practice consultations: is there any point being positive? BMJ 1987;294:1200-2.

8 Finniss DG, Kaptchuk TJ, Miller F, Benedetti F. Biological, clinical and ethical advances of placebo effects. Lancet 2010;375:686-95.

9 Harmden A, Lehman R. New primary care series: easily missed? BMJ 2009;338:b491.

10 Jones R, Charlton J, Latinovic R, Gulliford M. Alarm symptoms and identification of non-cancer diagnoses in primary care: cohort study. $B M$ 2009;339:b3094

11 Williamson P, Beitman BD, Katon W. Beliefs that foster physician avoidance of psychosocial aspects of health care. J Fam Pract 1981;13:999-1003.

12 Dowrick C, Gask L, Hughes JG, Charles-Jones H, Hogg JA, Peters S, et al. General practitioners' views on reattribution for patients with medically unexplained symptoms: a questionnaire and qualitative study. BMC Fam Pract 2008;19:46.

13 Kostopoulou O, Delaney BC, Munro CW. Diagnostic difficulty and error in primary care-a systematic review. Fam Pract 2008;25:400-12.

14 Kostopoulou O, Devereaux-Walsh C, Delaney BC. Missing celiac disease in family medicine: the importance of hypothesis generation. Med Decis Making 2009;29:282-90.

15 Salmon P, Dowrick CF, Ring A, Humphris GM. Voiced but unheard agendas: qualitative analysis of the psychosocial cues that patients with unexplained symptoms present to general practitioners. BrJ Gen Pract 2004;54:171-6.

16 Groopman J. How doctors think. Houghton Mifflin Company, 2007.

17 Salmon P, Ring A, Dowrick CF, Humphris GM. What do general practice patients want when they present medically unexplained symptoms, and why do their doctors feel pressurized? J Psychosom Res 2005;59:255-60.

18 Salmon P, Ring A, Humphris GM, Davies JC, Dowrick CF. Primary care consultations about medically unexplained symptoms: how do patients indicate what they want? J Gen Intern Med 2009;24:450-6.

19 Peters S, Rogers A, Salmon P, Gask L, Dowrick C, Towey M, et al. What do patients choose to tell their doctors? Qualitative analysis of potential barriers to reattributing medically unexplained symptoms. J Gen Intern Med 2009;24:540-2.

20 Salmon P, Humphris GM, Ring A, Davies JC, Dowrick CF. Primary care consultations about medically unexplained symptoms: patient presentations and doctor responses that influence the probability of somatic intervention. Psychosom Med 2007;69:571-7.

21 Hatcher S, Arroll B. Assessment and management of medically unexplained symptoms. BM/ 2008;336:1124-8.

22 Rosendal M, Bro F, Fink P, Christensen KS, Olesen F. Diagnosis of somatisation: effect of an educational intervention in a cluster randomised controlled trial. BrJ Gen Pract 2003;53:917-22.

\section{The wall of fame}

Below is a random sampling of remarkable investigation results, diagnostic studies, and experiences shared by our medicine residents over the past few years which appear on our "wall of fame." Our wall functions as a portal for the education, team building, and bonding that is core to the successful development and acculturation of young physicians. The remarkable facts on the wall pique curiosity and inspire a healthy competition, with our residents taking pride in raising the bar by updating or adding to its content. Highest fever: $109^{\circ} \mathrm{F}$

Highest blood pressure: 309/166

Most coronary care unit admissions in 24 hours: 17

Most codes [cardiac arrest calls] on the same patient during one shift: three

Highest number of cross coverage calls in 10 hours: 81

Lowest ionised calcium: $0.40 \mathrm{mmol} / \mathrm{l}$

Highest glucose on first day of internship: $2300 \mathrm{mg} / \mathrm{dl}$

Highest $\mathrm{PCO}_{2}: 170 \mathrm{~mm} \mathrm{Hg}$

Highest non-haemolysed potassium: $18 \mathrm{mmol} / 1$ "alive and kicking"

Highest NT-proBNP [brain natriuretic peptide]: 348000 pg $/ \mathrm{ml}$

Highest blood urea nitrogen: $354 \mathrm{mg} / \mathrm{dl}$
Lowest haemoglobin in an ambulatory patient: $1.6 \mathrm{~g} / \mathrm{dl}$

Highest sodium: $187 \mathrm{mmol} / \mathrm{l}$

Highest blood alcohol: $680 \mathrm{mg} / \mathrm{dl}$

Lowest temperature: $81^{\circ} \mathrm{F}$

Oldest patient: 107 years

Oldest surgical patient: 102 years

Highest INR: 33

Highest furosemide dose: $480 \mathrm{mg}$ daily

Lowest number of pages [bleeps] on a night float shift: three

Highest thyroid stimulating hormone: $743.60 \mathrm{mIU} / \mathrm{ml}$

Highest parathyroid hormone, intact: 1912 pg/ml

Highest ammonia level: 700 umole/1

Heaviest patient: $749 \mathrm{lb}$ [340 kg, $53 \mathrm{st} 7 \mathrm{lb}$ ]

Oldest patient requesting sildenafil: 85 years

Lowest high density lipoprotein: $5 \mathrm{mg} / \mathrm{dl}$

Highest white blood cell count: $629800 \times 10^{3} / \mathrm{mm}^{3}$

Highest H/H [haemoglobin and haematocrit]: $26.7 \mathrm{~g} / \mathrm{dl} / 75.3 \%$

Longest QTc interval on electrocardiogram: $625 \mathrm{~ms}$

Furthest discharge destination: Ukraine.

Michael J Rosenblum medical director, Baystate Medical Center,

Springfield, USA michael.rosenblum@bhs.org

Cite this as: BMJ 2010;340:c421 